\title{
Kôm Ombo (2019)
}

\section{Françoise Labrique et Aurélie Terrier}

\section{OpenEdition \\ Journals}

Édition électronique

URL : https://journals.openedition.org/baefe/1053

DOI : $10.4000 /$ baefe. 1053

ISSN : 2732-687X

Éditeur

ResEFE

\section{Référence électronique}

Françoise Labrique, Shafia Bedier, Aurélie Terrier, « Kôm Ombo (2019) » [notice archéologique], Bulletin archéologique des Écoles françaises à l'étranger [En ligne], Égypte, mis en ligne le 05 octobre 2020, consulté le 28 juillet 2021. URL : http://journals.openedition.org/baefe/1053 ; DOI : https://doi.org/ $10.4000 /$ baefe. 1053

Ce document a été généré automatiquement le 28 juillet 2021.

\section{cc) (†) $\odot$}

Le Bulletin archéologique des Écoles françaises à l'étranger est mise à disposition selon les termes de la Licence Creative Commons Attribution - Pas d'Utilisation Commerciale - Pas de Modification 4.0 International. 


\title{
Kôm Ombo (2019)
}

\author{
Françoise Labrique et Aurélie Terrier
}

\section{NOTE DE L'AUTEUR}

Année de la campagne : 2019 ( $1^{\text {er }}$ octobre -16 octobre) Numéro et intitulé de l'opération de terrain : 17151 - Le temple de Haroéris et Sobek à Kôm Ombo : relevés photographiques, mission épigraphique, préservation du site Composition de l'équipe de terrain : L'équipe était composée de Françoise Labrique (épigraphiste, Universität zu Köln), Shafia Bedier (épigraphiste, université Ayn Shams), Ali Abdelhalim (épigraphiste, concepteur des banquettes et du proto-musée épigraphique, université Ayn Shams, Ifao), Islam Alwakeel (épigraphiste, université Ayn Shams), Anna Dékány (épigraphiste, Universität zu Köln), Svenja Dirksen (dessinatrice, Universität Würzburg), Sven Eicke (épigraphiste, Universität zu Köln), Mohamed Gaber (topographe, Ifao), Emmanuel Laroze (architecte, CNRS), Nicolas Leroux (épigraphiste, FNRS), Sara Nabil (épigraphiste, université Ayn Shams), Gaël Pollin (photographe, Ifao), Aurélie Terrier (architecte-archéologue, CNRS, IRAA USR3155, Université de Genève), Janine Traber (dessinatrice, Universität zu Köln) et Mohamed Abdou (intendant de chantier, Ifao). Le ministère des Antiquités était représenté par l'inspecteur Essam Mahmoud et le restaurateur Mohamed Abdelrahim

Partenariats institutionnels : La mission bénéficie du soutien de Universität zu Köln, de l'université Ayn Shams et du ministère des Antiquités.

Sponsors : Deutsche Forschungsgemeinschaft

\section{Problématique et bilan des principaux résultats}

1 La mission poursuit les travaux épigraphiques qui avaient été interrompus par le décès d'Adolphe Gutbub. Actuellement, un volume est en préparation, consacré à la petite salle hypostyle (PM VI, 186, salle B), dont la couverture photographique est confiée à Gaël Pollin. Au projet épigraphique relancé en 2010 s'ajoutent à présent deux points : construire, à la demande du ministère des Antiquités (MoA), des socles destinés à 
exposer les blocs restés épars dans le secteur au nord du temple; et commencer une étude architecturale du monument.

\section{Mission d'octobre 2019 au temple de Kôm Ombo}

2 - Mastabas et dépôt lapidaire. Le travail est étroitement suivi par Ali Abdelhalim, qui a défini le projet et sa réalisation en relation avec l'inspectorat et a conçu et soumis au MoA un modèle de distribution et d'exposition des blocs. Le site d'implantation choisi par l'inspectorat consiste en une grande cavité archéologique. L'intendant de chantier Mohamed Abdou a engagé des spécialistes pour la construction, qui s'est opérée du 2 au 9 octobre.

Spécialistes : Mohamed Hussein Abdallah; Azeb Hussein Mohamed; Mohamed Ahmed Kaï ; Mohamed Mahmoud Mohamed Shiemy.

Quatre mastabas de longueur inégale ont été construits durant cette première campagne.

Fig. 1. État avant travaux.

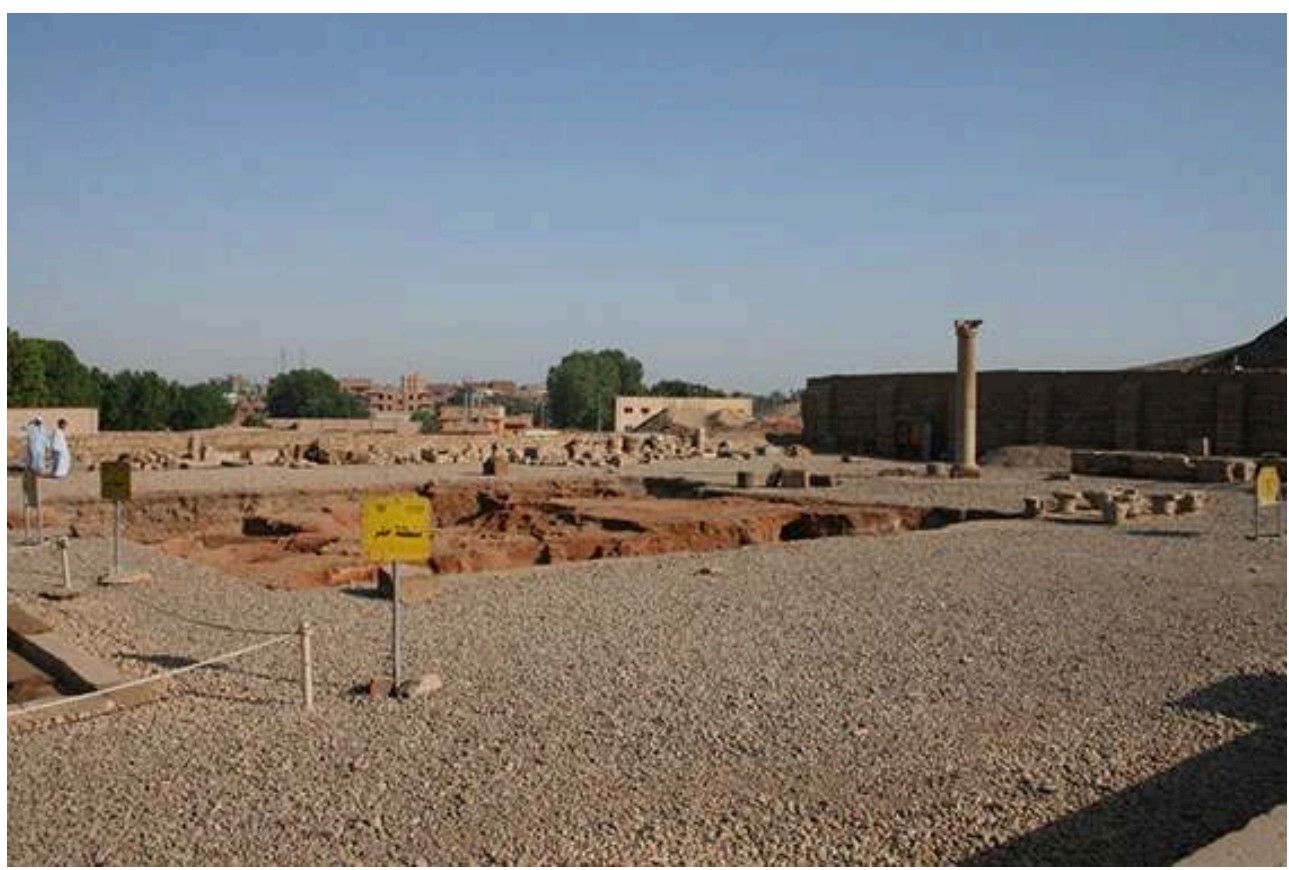

Mission 2019_4438.

(C) Ifao. 17151_2019_NDMPF_001 
Fig. 2. Ébauche de mise en place.

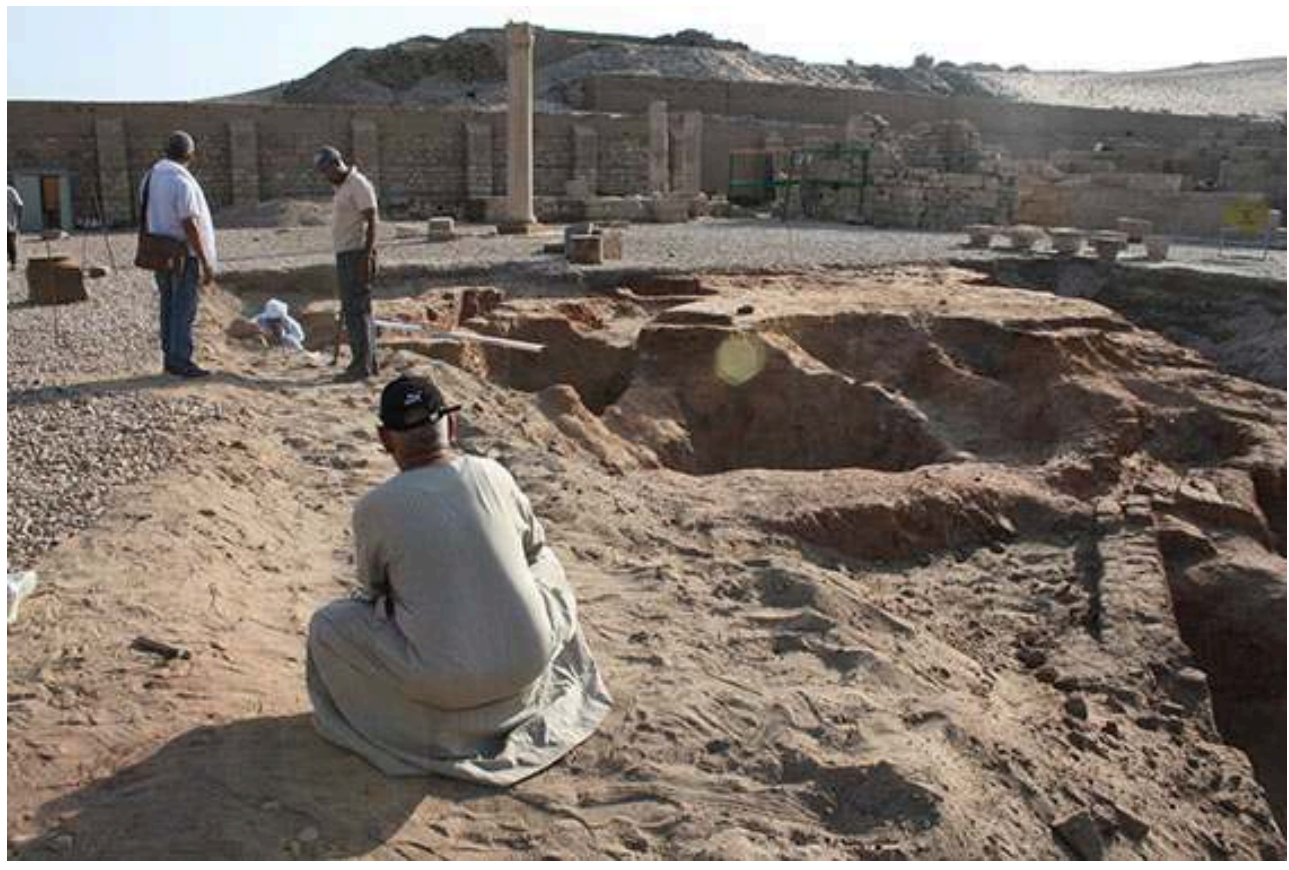

Mission 2019_4501.

(C) Ifao. 17151_2019_NDMPF_002

Fig. 3. Halage.

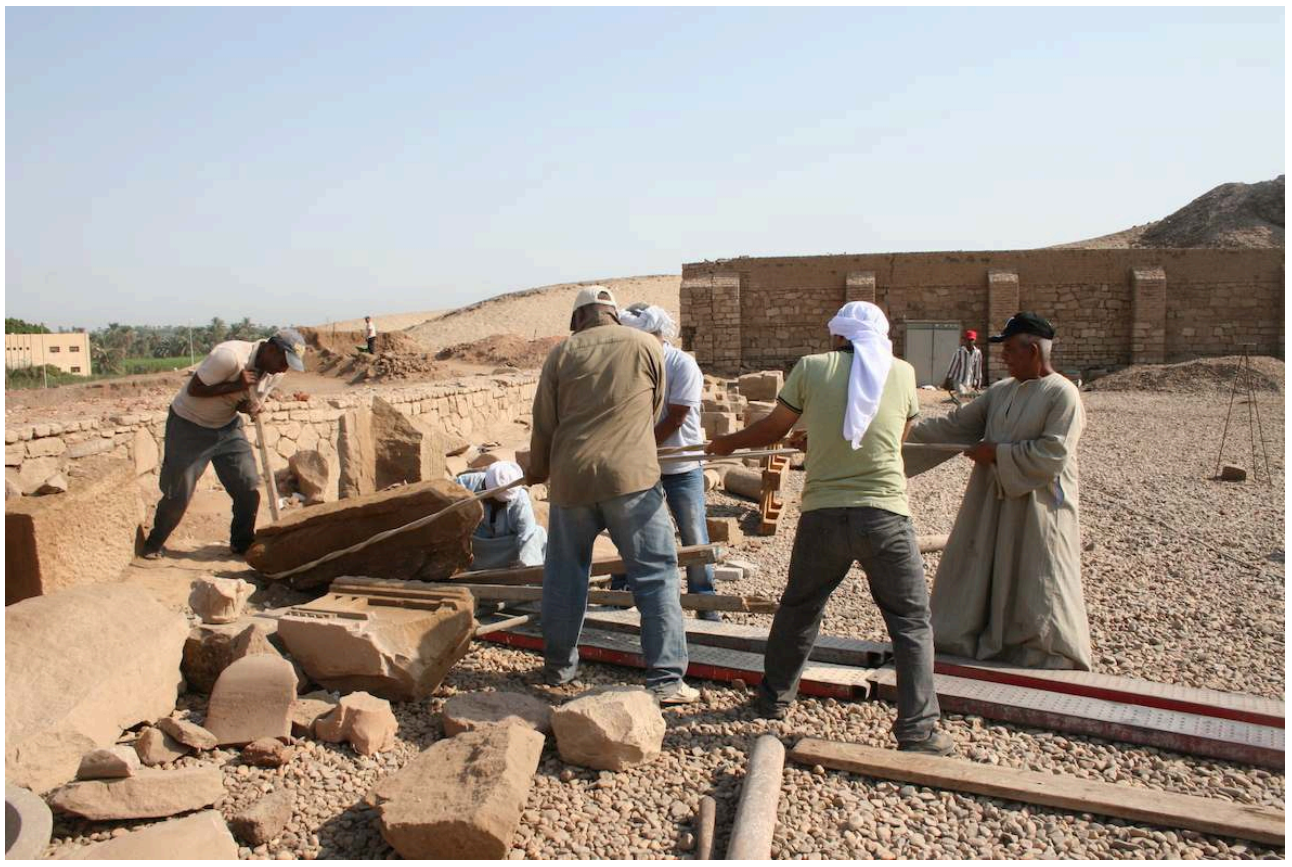

Mission 2019_5731.

(c) Ifao. 17151_2019_NDMPF_003 
Fig. 4. Une statue de lion a été mise en place.

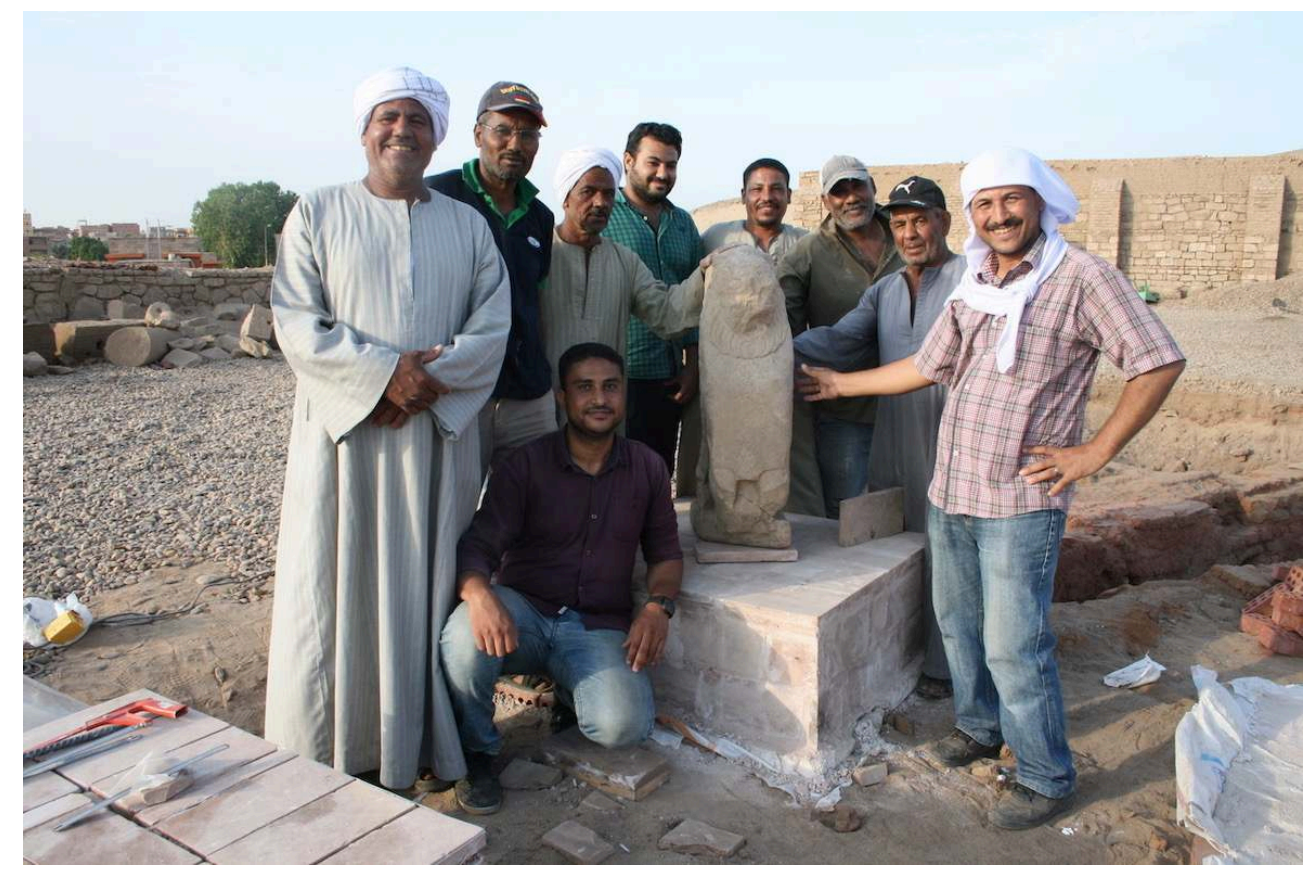

Mission 2019_5072.

(c) Ifao. 17151_2019_NDMPE_001

3 Plusieurs pierres lourdes portant des inscriptions ont été placées sur les autres mastabas en attendant d'être disposées selon un programme établi. Une banquette est réservée aux tambours et chapiteaux de colonne. Une corde a été placée autour de l'ensemble pour en interdire l'accès jusqu'à l'année suivante. Les matériaux inutilisés ont été rassemblés en une banquette factice jusqu'à la reprise des travaux. 
Fig. 5. État provisoire du musée lapidaire.

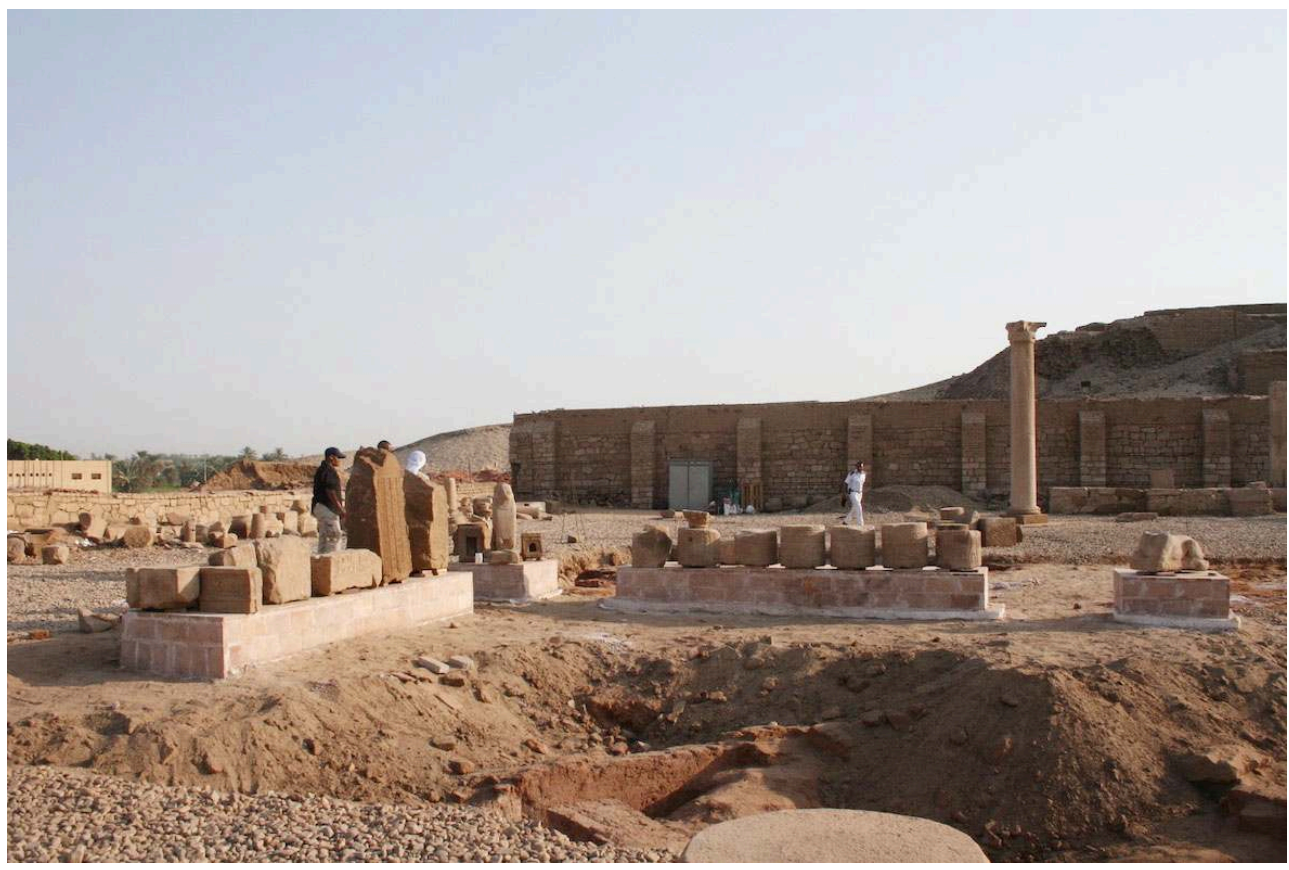

Mission 2019_5908.

(c) Ifao. 17151_2019_NDMPF_004

Fig. 6. Une corde est tendue autour du site.

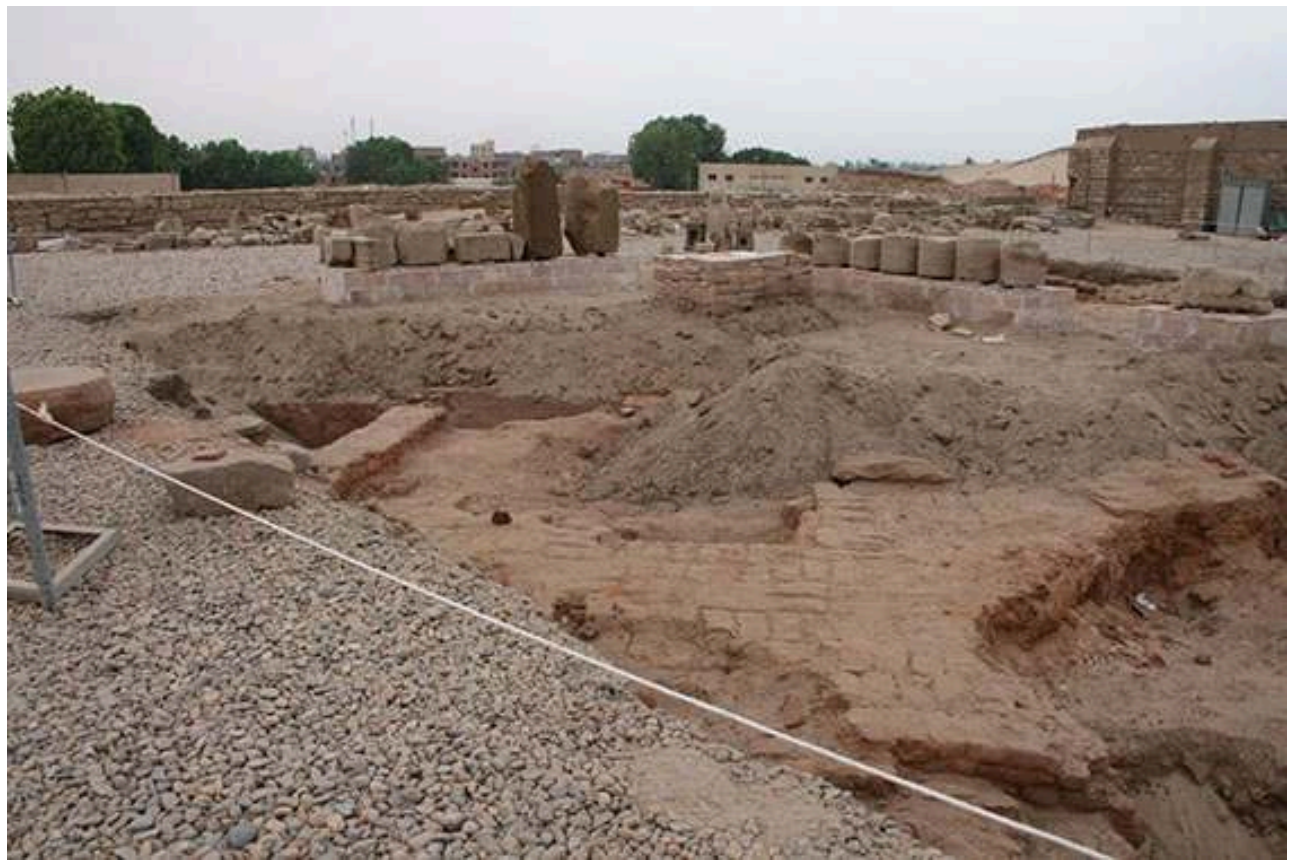

Mission 2019_7195.

(C) Ifao. 17151_2019_NDMPF_005

4 Les directeurs des inspectorats de Kôm Ombo et d'Assouan ont exprimé leur grande satisfaction pour ce soutien dans l'aménagement et la protection du site. 
5 - Travail épigraphique. L'équipe, constituée de Ali Abdelhalim, Islam Alwakeel, Anna Dékány, Svenja Dirksen, Sven Eicke, Françoise Labrique, Janine Traber, a collationné les relevés réalisés depuis la mission 2018 dans la petite salle hypostyle.

6 - G. Pollin a achevé la couverture photographique de la petite salle hypostyle: les abaques, architraves et trois colonnes, ensemble qui réclame une manipulation virtuose des échafaudages. Il a ajouté à cette documentation la scène au relief chirurgical et les tableaux axiaux du couloir externe est.

7 Avec l'aide d'Emmanuel Laroze, il a également réalisé un test sur une colonne du pronaos, en prévision de la mission de 2020.

8 - Graffiti. Sara Nabil (démotisante) a repéré un graffito hiératique et plusieurs graffiti démotiques inédits. Leur étude est désormais en cours.

9 Ali Abdelhalim a complété pour sa part ses propres relevés de graffiti animaliers. Aurélie Terrier, en compagnie d'Islam Alwakeel et de l'inspecteur Essam Mahmoud, a découvert des graffiti supplémentaires sur le toit.

10 - Formation. Sous la direction de S. Dirksen, Islam Alwakeel s'est initié au dessin sur table graphique à l'aide d'Adobe Illustrator. J. Traber s'est quant à elle initiée aux relevés architecturaux sous la férule d'A. Terrier.

11 - Étude architecturale et archéologique. A. Terrier est depuis cette année membre de la mission en tant que responsable de l'étude architecturale et archéologique du temple. Financée par la Maison de l'histoire de l'Université de Genève, elle avait cette année à mettre en place la nomenclature et la méthodologie pour les futures études et relevés. En vue des travaux qui débuteront dès l'année prochaine, un réseau de stations a été installé, ce qui a permis un relevé au tachéomètre des deux axes de circulation, de deux des quatre couloirs latéraux d'axe est-ouest (déambulatoires intérieurs et extérieurs) et des gabarits principaux des espaces du temples (naos, pronaos et cour). Ce travail a été réalisé avec la collaboration du topographe Mohamed Gaber. Parallèlement, A. Terrier a procédé au relevé systématique des marques de carrier présentes en plusieurs endroits du temple ainsi qu'aux mortaises conservées. A. Terrier a également pris des mesures pour les gabarits généraux des murs de la salle hypostyle en vue de la publication des textes dans Kôm Ombo III. Enfin, en prévision de l'arrivée de la production pour l'émission de RMC découverte le 23 octobre, A. Terrier a mis en place les cibles pour la photogrammétrie des murs intérieurs ouest de la cella. Pour sa part, E. Laroze, prenant des mesures et continuant ses observations dans le mammisi, y a procédé aux relevés photogrammétriques.

12 Il est prévu que Mohamed Gaber séjourne deux semaines entières à Kôm Ombo en 2020 pour réaliser le plan topographique général du site.

13 - Nicolas Leroux a été hôte de la mission cette année pour son projet personnel de collationnement des hymnes-senedj dans le temple majeur. Son séjour est financé par le FNRS (Communauté française de Belgique). Le collationnement est achevé.

\section{Publications}

14 Un volume consacré à la salle médiane ( $\mathrm{PM} \mathrm{VI}, 186$, salle C) ainsi qu'à ses annexes latérales (chambre d'introduction des offrandes, chambre de l'inondation, laboratoire) 
a été déposé à l'Ifao, où il paraîtra sous le titre Kom Ombo II, avec pour auteurs Shafia Bedier, Françoise Labrique, Ali Abdelhalim, Anna Dékány, Sven Eicke.

15 Ali Abdelhalim, "Notes on the Bandeau-Texts of Columns of Kom Ombo Temple ", BCPS 36, 2019, p. 287-304

16 Françoise Labrique, Gaël Pollin, "Kôm Ombo », in Laurent Coulon, Mélanie Cressent (éd.), Archéologie française en Égypte, BiGen 59, Le Caire, 2019, p. 182-187.

\section{Valorisation de la recherche}

17 Conférence de Françoise Labrique dans le cadre d'une Journée d'étude organisée par René Preys, Les temples d'époque gréco-romaine à l'Université de Namur, le 10 mai 2019 : «Le projet Kom Ombo ».

\section{INDEX}

Année de l'opération : 2019

lieux https://ark.frantiq.fr/ark:/26678/pcrt3UJQm0Xkzv sujets https://ark.frantiq.fr/ark:/26678/pcrt30jRVudWjq, https://ark.frantiq.fr/ark:/26678/ pcrtd1J2aYQ6GE, https://ark.frantiq.fr/ark:/26678/pcrtJVKn9XsZH9, 26678/pcrtvun1g7PPLq, https://ark.frantiq.fr/ark:/26678/pcrtJrInD7k8G1, https://ark.frantiq.fr/ark:/26678/ pcrtIgoNn37WDP, https://ark.frantiq.fr/ark:/26678/pcrtF9P4mbuyGk, https://ark.frantiq.fr/ ark:/26678/pcrtKa92NyYWRf, https://ark.frantiq.fr/ark:/26678/pcrt2m165SIOlQ

oeuvres https://ark.frantiq.fr/ark:/26678/pcrtRt1cNzZgPS

Thèmes : IFAO

\section{AUTEURS}

FRANÇOISE LABRIQUE

Épigraphiste, Universität zu Köln

\section{AURÉLIE TERRIER}

Architecte-archéologue, CNRS, IRAA USR3155, Université de Genève 